\title{
LESSON 125
}

\section{Combination Signs}

Type Combination Signs

PAPER: A4.

MARGINS: Pica, 15-67; Elite, Sign 25-77.

TABS: Pica 20; Elite 30.

Use single-line spacing with a blank line between each item.

Type the instructions and then practise the sign in the wide left margin.

Keep the sheet for reference.
Although most typewriters have a standard range of keys, there are certain keys which may not be provided, as, for example, the figures one and nought. Below is a list which contains several keys you may not have. Even if you do have the keys it is suggested that you type out the exercise in case your next machine does not have them.

\section{COMBINATION SIGNS}

How it is made

The exclamation mark is produced by typing the apostrophe and backspacing before typing the full stop.

The asterisk - turn the roller anti-clockwise one 'click', type the hyphen, backspace and type a

* small x.

The division sign is produced by typing a colon and backspacing before typing the hyphen.

The multiplication sign is simply a small $\mathbf{x}$. The minus sign is simply a hyphen.

The equals sign is the hyphen typed twice - using the interliner and the backspacer.

The dollar sign is produced by typing a capital $S$ $\$$ and backspacing before typing the solidus over it. The dagger is used in literary work to indicate an explanatory footnote. Type a capital $I$ and

I backspace before typing the hyphen over it.

The double dagger is produced by typing a capital I. Backspace and use the interliner to turn the paper up slightly before typing another 王 capital I over the first.

Type the solidus. Backspace and type the underscore. It should look like this L Turn down the roller two 'clicks' if your machine has the half line space, or one 'click' if it does not, and type the underscore again. This sign is called a square bracket.

To produce the other bracket, type the underscore and then the solidus. Backspace and turn the paper down two 'clicks' if your machine has the half line space, or one 'click' if it does not, and type in

T the underscore again.

4' The abbreviation for feet is the apostrophe.

$6 "$ The abbreviation for inches is the quotation marks. 